\title{
Pharmacological Selectivity Within Class I Histone Deacetylases Predicts Effects on Synaptic Function and Memory Rescue
}

\author{
Gavin Rumbaugh", ${ }^{*, 5}$, Stephanie E Sillivan ${ }^{1,2,5}$, Emin D Ozkan', Camilo S Rojas', Christopher R Hubbs', \\ Massimiliano Aceti', Mark Kilgore ${ }^{3}$, Shashi Kudugunti ${ }^{4}$, Sathyanarayanan V Puthanveettil', J David Sweatt', \\ James Rusche ${ }^{4}$ and Courtney A Miller, ${ }^{*, 1,2}$
}

'Department of Neuroscience, The Scripps Research Institute, Jupiter, FL, USA; ${ }^{2}$ Department of Metabolism and Aging, The Scripps Research Institute, Jupiter, FL, USA; ${ }^{3}$ Department of Neurobiology, The Evelyn F. McKnight Brain Institute, The University of Alabama at Birmingham, Birmingham, AL, USA; ${ }^{4}$ The Repligen Corporation, Waltham, MA, USA

\begin{abstract}
Histone deacetylases (HDACs) are promising therapeutic targets for neurological and psychiatric disorders that impact cognitive ability, but the relationship between various HDAC isoforms and cognitive improvement is poorly understood, particularly in mouse models of memory impairment. A goal shared by many is to develop HDAC inhibitors with increased isoform selectivity in order to reduce unwanted side effects, while retaining procognitive effects. However, studies addressing this tack at the molecular, cellular and behavioral level are limited. Therefore, we interrogated the biological effects of class I HDAC inhibitors with varying selectivity and assessed a subset of these compounds for their ability to regulate transcriptional activity, synaptic function and memory. The HDAC- I, -2, and -3 inhibitors, RGFP963 and RGFP968, were most effective at stimulating synaptogenesis, while the selective HDAC3 inhibitor, RGFP966, with known memory enhancing abilities, had minimal impact. Furthermore, RGFP963 increased hippocampal spine density, while HDAC3 inhibition was ineffective. Genome-wide gene expression analysis by RNA sequencing indicated that RGFP963 and RGFP966 induce largely distinct transcriptional profiles in the dorsal hippocampus of mature mice. The results of bioinformatic analyses were consistent with RGFP963 inducing a transcriptional program that enhances synaptic efficacy. Finally, RGFP963, but not RGFP966, rescued memory in a mouse model of Alzheimer's Disease. Together, these studies suggest that the specific memory promoting properties of class I HDAC inhibitors may depend on isoform selectivity and that certain pathological brain states may be more receptive to HDAC inhibitors that improve network function by enhancing synapse efficacy.

Neuropsychopharmacology (2015) 40, 2307-23I6; doi:I0.1038/npp.20I5.93; published online 22 April 20I5
\end{abstract}

\section{INTRODUCTION}

Manipulation of gene transcription through epigenetic modifications to DNA and chromatin represents a promising and broad therapeutic avenue for the treatment of central nervous system disorders, including disorders of cognitive ability, such as Alzheimer's Disease (AD). AD is marked by increasing dementia as neurodegeneration progresses, starting with the loss of synapses (Terry et al, 1991), which are fundamental components of the brain's memory storage system. Epigenetics has received particular attention in recent years for its role in memory (Day and Sweatt, 2011), and hopes are particularly high for the potential of epigenetic

* Correspondence: G Rumbaugh or Dr CA Miller, Department of Metabolism and Aging, The Scripps Research Institute, I30 Scripps Way, Jupiter, FL 33458, USA, Tel/Fax: + | 561 228346 I or + | 561228 2958, E-mail: grumbaug@scripps.edu or cmiller@scripps.edu

${ }^{5}$ These authors contributed equally to this work.

Received II December 2014; revised 25 March 2015; accepted 31

March 2015; accepted article preview online 3 April 2015 modulation to drive cognitive enhancement and, perhaps, to rescue memory in AD (Abel and Zukin, 2008; Mikaelsson and Miller, 2011; Rudenko and Tsai, 2014).

Chromatin's core histone proteins undergo posttranslational modification with memory, including acetylation, providing some molecular clues to how these enzymes regulate cognition. Transcriptionally permissive histone acetylation is regulated by histone acetyltransferases, which add acetyl moieties, and histone deacetylases (HDACs), which remove them. There are five classes of transcriptionally repressive HDACs. Class I (HDAC1, $-2,-3$, and -8 ), class IIA (HDAC4, -5, -7, and -9), class IIB (HDAC6 and -10), and class IV (HDAC11) are zinc dependent, whereas class III sirtuins (SIRT1-7) are NAD-dependent and can also participate in non-histone protein acetylation. Numerous laboratories have now demonstrated that pharmacological inhibition of HDACs, which leads to an accumulation of lysine acetylation and transcriptional activation, is capable of modulating memory (Graff and Tsai, 2013; Levenson et al, 2004). This has driven an excitement in the field for the potential of memory rescue in the face of disorders 
characterized by widespread synapse loss and memory impairments. However, the 11 non-sirtuin HDACs are involved in a myriad of processes throughout the body, leading many to propose that increasing HDACi isoform selectivity may improve specificity for a given brain disorder and simultaneously decrease unwanted off-target effects (Graff and Tsai, 2013; Haggarty and Tsai, 2011; Mikaelsson and Miller, 2011). This naturally begs the question of which isoform(s) represent the ideal target for treating memory disorders.

Class I HDACs have received the most attention in relation to cognition. For instance, genetic and embryonic deletion of HDAC2, but not HDAC1, was found to enhance memory in wild-type (WT) mice, and furthermore viralmediated knockdown of HDAC2 in the hippocampal CA1 region was sufficient to rescue memory in a mouse model of neurodegeneration (Graff et al, 2012; Guan et al, 2009). Similar memory enhancement was found following postnatal forebrain deletion of HDAC2 using a CAMKII-Cre driver (Morris et al, 2013). Likewise, focal deletion of HDAC3 accomplished through viral delivery of Cre into the hippocampus of healthy adult HDAC3-floxed mice enhanced memory (McQuown et al, 2011), although the role of this isoform has not been tested in mouse models of cognitive dysfunction. While broad genetic deletion of an HDAC may be accompanied by various developmental compensations and is therapeutically impractical, more temporally restricted manipulations of HDAC activity can be achieved through pharmacological targeting with small molecules. These manipulations may also be more specific compared with genetic deletion because they target enzymatic activity, rather than deleting the protein. There are at least three classes of engineered HDACi's: carboxylic acids (eg, sodium butyrate $(\mathrm{NaB})$ and sodium phenylbutryate (SPB)), hydroxamic acids (eg, trichostatin A (TSA), suberoylanilide hydroxamic acid (SAHA), and crebinostat), and ortho-aminoanilines or benzamides (eg, RGFP136). However, a pharmacological approach to HDAC inhibition has historically meant sacrificing isoform selectivity. For instance, TSA, which can enhance memory when delivered to a healthy brain (Blank et al, 2014; Fischer et al, 2007; Hawk et al, 2011; Monsey et al, 2011), targets all 11 HDACs. SPB and valproic acid, HDACi's with similarly broad activity, rescue memory in mouse models of $\mathrm{AD}$ (Kilgore et al, 2010; Ricobaraza et al, 2009). Additional insight into the role of individual HDACs in cognition can be gleaned from HDACi's with greater isoform specificity. For example, the most commonly used HDACi for cognitive studies, NaB, was once thought to be a broad spectrum inhibitor, but has since been determined to have a surprising degree of selectivity for class I HDACs (Kilgore et al, 2010). Further, SAHA and crebinostat's activity are largely limited to class I HDACs $(-1$, $-2,-3$, and -8) and HDAC6. Acute administration of $\mathrm{NaB}$, SAHA, and crebinostat have all been shown to enhance memory in WT mice and to drive synaptogenesis (Blank et al, 2014; Fass et al, 2013; Fischer et al, 2007; Guan et al, 2009; Intlekofer et al, 2013; Levenson et al, 2004; Mahan et al, 2012). Further, $\mathrm{NaB}$ and SAHA rescue memory in mice with reduced cognitive ability, including $\mathrm{AD}$ models (Fischer et al, 2007; Kilgore et al, 2010). Two of the most selective HDACi's used to date, RGFP136 and RGFP966, bear a high degree of selectivity for HDAC3 and enhance memory in WT mice
(Malvaez et al, 2013; McQuown et al, 2011). When the results of these studies are taken together, it can readily be concluded that manipulating members of class I HDACs $(-1$, $-2,-3$, and -8) can improve memory, with genetic manipulations further pointing to the potential of HDAC2 and -3 as targets for improving cognition in a 'diseased' state. However, selective inhibitors of HDAC2 or -3 have not been tested in mouse models of reduced cognitive function. Furthermore, the impact of selective inhibitors on synaptic function and spinogenesis, which are the neurobiological substrates believed to drive improved cognition in $\mathrm{AD}$ models (Knobloch and Mansuy, 2008; Nistico et al, 2012; Pozueta et al, 2013), remains unknown. Therefore, we assessed the impact of several new HDACi's with differing class I selectivity profiles at various neurobiological levels to better understand the relationship between HDACi isoform selectivity and regulation of cognitive function.

\section{MATERIALS AND METHODS}

For additional details, please see Supplementary Materials and Methods.

\section{Animals}

Eight-week-old C57BL/6 mice were used for gene expression analyses (The Jackson Laboratory, Bar Harbor, ME). Thy1-GFP(m) (B6.Cg-Tg(Thy1-EGFP)MJrs/J; stock number 007788) and APPswe/PS1dE9 double-transgenic mice (APP/ PS1; B6C3-Tg (APPswe,PSEN1dE9)85Dbo/J; stock number 004462) (Jankowsky et al, 2003) were also obtained from Jackson Laboratory. P0 CD1 mice were used for electrophysiological recordings and synaptogenesis assays (CD1 (ICR) (strain code 022), Charles River Laboratories, Hollister, CA). All procedures were performed in accordance with the Scripps Florida Research Institute at Jupiter, Florida Institutional Animal Care and Use Committee and with national regulations and policies.

\section{Drugs}

TSA (Tocris) was dissolved in DMSO at a concentration of 300 mM. RGFP966, RGFP963, and RGFP233 were provided by Repligen Corporation. The drugs were dissolved in DMSO and diluted in 30\% HPBCD and $0.1 \mathrm{M}$ acetate, $\mathrm{pH}$ 5.5. The final DMSO concentration was no $>5 \%$ and the same concentration of DMSO was used in the vehicle. For in vivo experiments, drugs were administered at $30 \mathrm{mg} / \mathrm{kg}$ IP.

\section{Gene Expression Profiling}

RNA sequencing was performed on $100 \mathrm{ng}$ of hippocampal RNA obtained from mice after 3 days of daily, systemic injection of RGFP963, RGFP966, or vehicle. Results were validated with quantitative PCR (qPCR) using standard Taqman assays (Life Technologies).

\section{Synaptogenesis Assay}

Primary cortical neuron cultures were prepared from cortices dissected from P0 CD1 mice. Neurons were then seeded onto 96-well imaging plates (Corning) and 
were transfected with synaptophysin (SYN)-YFP plasmid constructs. ImageJ software (NIH) was used to quantify the relative number of fluorescent puncta obtained by imaging with the InCell 6,000 at DIV10, relative to DIV8.

\section{Electrophysiology}

Whole-cell voltage clamp experiments were performed on pyramidal neurons from P0 hippocampal cultures. Drugs were added at DIV4 and recordings were made $18-24 \mathrm{~h}$ later. Miniature excitatory postsynaptic currents (mEPSCs) were isolated with $50 \mu \mathrm{M}$ picrotoxin, $1 \mu \mathrm{M}$ tetrodotoxin and $50 \mu \mathrm{M}$ DL-AP5 (Tocris), and recorded at $-70 \mathrm{mV}$. Analysis of miniature events was performed using Clampfit 10.4 software (Molecular Devices).

\section{In Vivo Spine Imaging and Quantification}

After 12 daily IP drug treatments, 11-week-old Thy1-GFP (m) mice were deeply anesthetized and perfused transcardially. Tissue was mounted onto microscope slides with an antifade reagent with DAPI (Prolong Gold, Life Technologies). For spine density and morphology, multiple branches emanating from 6-10 dendritic segments of eGFP+ CA1 oblique branches of pyramidal neurons that were $\sim 20-30 \mu \mathrm{m}$ in length were imaged by Z-sectioning through the slice (from 25 to $75 \mu \mathrm{m}$ from slice surface). Images were fast filtered with Image J. As segments were traced, each individual spine was marked. Only protuberances with a clear connection of the head of the spine to the dendritic shaft were counted as a spine. Pictures were visualized and elaborated with Neurolucida software (MicroBrightField).

\section{Contextual Fear Conditioning}

APP/PSI mice at least 6 months of age were given 12 daily IP injections of the appropriate compound or vehicle. At least $24 \mathrm{~h}$ after the final injection, mice were placed into the training chamber and allowed to explore for $2.5 \mathrm{~min}$, after which they received a single electric foot shock $(2 \mathrm{~s}$, $0.75 \mathrm{~mA}$ ). After the shock, the mice remained in the chamber for an additional $28 \mathrm{~s}$ before being returned to their home cage. To test for long-term memory, freezing was assessed $24 \mathrm{~h}$ later during a 5 -min exposure to the context.

\section{Statistical Analyses}

One- or two-way univariate ANOVAs were applied to all data. Student's $t$-tests and post hoc tests were used when necessary. Significance was set at $P<0.05$ for all tests. All analyses were performed blind with respect to treatment condition.

\section{RESULTS}

Pimelic diphenylamides selectively target class I HDACs with little modulation of other isoforms, and derivatives with selectivity for $\mathrm{HDAC} 1$ and -3 have shown efficacy in models of Freidriech's Ataxia and Huntington's Disease (Herman et al, 2006; Jia et al, 2012; Thomas et al, 2008). Unlike other classes of HDAC inhibitors, such as the hydroxymates (eg, SAHA), pimelic diphenylamides are competitive tight- binding inhibitors characterized by slow-on/slow-off kinetics, leading to persistent histone hyperacetylation in brain tissue (Rai et al, 2008). Two novel compounds, RGFP968 and RGFP963, have high potency for HDAC1, -2 , and -3 , with little to no activity against the remaining eight non-sirtuin HDACs (Figure 1a-d, Supplementary Table S1). In contrast, RGFP966 is $>200$-fold more selective for HDAC3 than -1 and -2 , with no activity detected at the other HDACs (Supplementary Table S1; Malvaez et al, 2013). We also investigated RGFP233, an inhibitor that targets HDAC1, $-2,-3$, and -10 , but with a $\sim 75$-fold greater selectivity for HDAC1 and -2 (Supplementary Table S1). Using this panel of HDAC-targeting compounds, we investigated how differing inhibition of HDAC isoforms impacted features of neuronal function, as well as memory in the context of a mouse model of AD.

To date, $\mathrm{NaB}$ represents the most selective HDACi (HDAC1, $-2,-3$, and -8) known to be capable of driving synaptogenesis. Therefore, we first determined the impact of HDAC inhibition limited to HDAC1, -2, and -3 (RGFP963 and RGFP968), HDAC1 and -2 (RGFP233), or HDAC3 alone (RGFP966) on synaptogenesis. Expression of YFP-tagged SYN in neurons reliably reports the presence of functional presynaptic terminals in cultured neurons (Harms et al, 2005). Therefore, we developed a live-imaging synaptogenesis assay based on this reporter construct. We expressed the SYN-YFP fusion in young cortical neurons and then confirmed that the axon-localized bright puncta colocalized with endogenous proteins expressed in synaptic vesicles (Figure 2a). These data suggested that counting the number of SYN-YFP+ structures over time on a per-neuron basis would provide a dynamic readout of synaptogenesis in developing cultured neurons. Based on this initial result, we designed an assay where neurons were imaged during a baseline session, and then vehicle or the broad spectrum HDACi TSA was added to individual culture wells. Two days later, the same wells were reimaged and the relative change in SYN-YFP+ structures was calculated (Figure $2 \mathrm{~b}$ and c). There was an increase of $\sim 15 \%$ in SYN-YFP+ puncta from DIV8 to DIV10 in the DMSO-treated wells. TSA-treated wells had a significantly greater increase over the same period $\left(\sim 50 \% ; t_{(10)}=-6.92, P<4.1 \mathrm{E}-05\right)$ (Figure $\left.2 \mathrm{~d}\right)$. This TSA-induced increase is consistent with a prior study that immunostained for changes in endogenous presynaptic protein puncta following TSA treatment (Akhtar et al, 2009; Shi et al, 2011). We next tested the RGFP compounds. The HDAC1, -2, and -3 inhibitor, RGFP963, produced robust, dose-dependent increases in synaptogenesis that were equivalent to TSA $\left(\mathrm{F}_{(3,20)}=105.12, P<2 \mathrm{E}-12\right.$; Figure $2 \mathrm{e}$ and $h)$. Similarly, the other HDAC1, -2 , and -3 inhibitor, RGFP968, produced significant synaptogenesis relative to DMSO control $\left(\mathrm{F}_{(5,30)}=57.12, P<1 \mathrm{E}-14\right.$; Figure $\left.2 \mathrm{~h}\right)$. However, the RGFP compounds with greater selectivity within class I produced less or no synaptogenesis (Figure $2 \mathrm{f}-\mathrm{h}$ ). For instance, the HDAC3-selective inhibitor, RGFP966, had minimal impact at the highest dose on the number of SYN-YFP+ structures relative to DMSO $\left(\mathrm{F}_{(3,20)}=4.85\right.$, $P<0.05$; Figure 2f), and no effect at lower doses. This minimal synaptogenesis did not replicate when tested again, relative to all four compounds and TSA $(P>0.05$; Figure $2 \mathrm{~h})$. And, while RGFP233, which preferentially targets HDAC1 and -2 , with less activity at HDAC 3 and -10 , produced an 
a

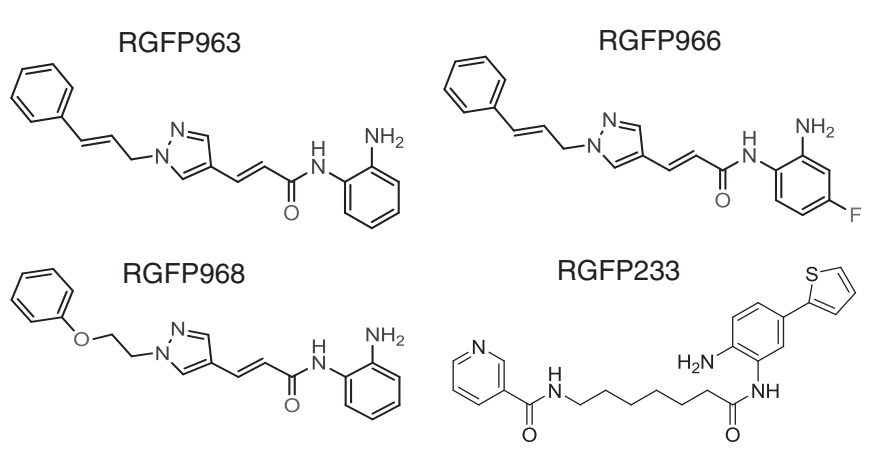

b

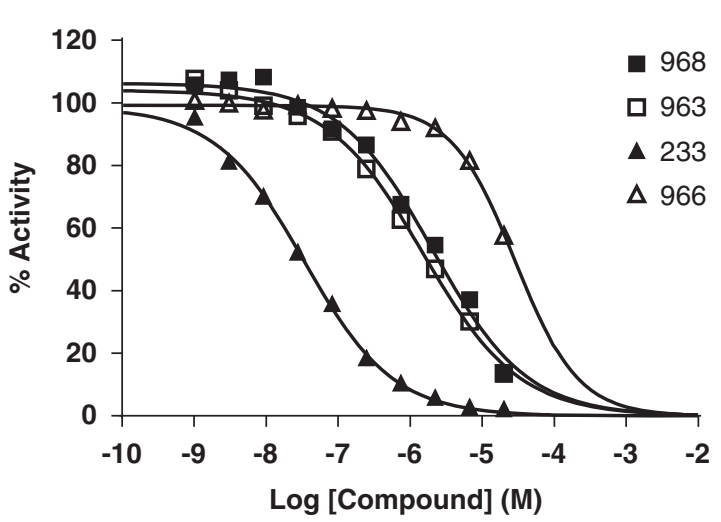

c Compound IC50 Data for HDAC2

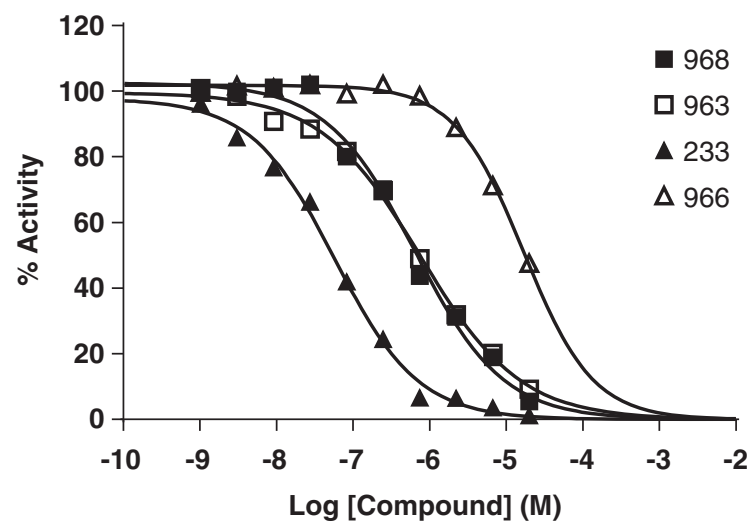

d

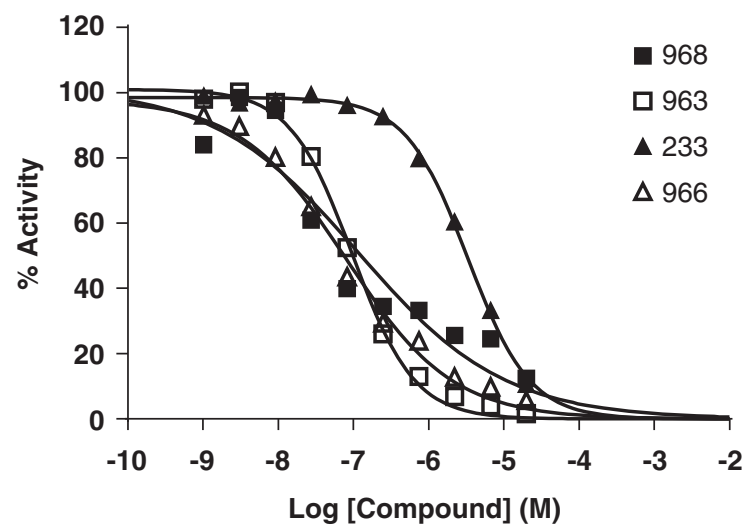

Figure I Novel pimelic diphenylamide-based histone deacetylase (HDAC) inhibitors have differential selectivity within the class I HDACs. (a) Chemical structures of novel RGFP HDAC inhibitors. IC 5 values for (b) HDACI, (c) HDAC2, and (d) HDAC3. See Supplementary Table SI for all other HDACs.

increase in SYN-YFP+ puncta at the highest dose tested $\left(\mathrm{F}_{(3,20)}=20.13, P<3 \mathrm{E}-6\right.$; Figure $\left.2 \mathrm{~g}\right)$, its impact was less than that of the HDAC1, -2 , and -3 inhibitors $(P<0.05$; Figure $2 \mathrm{~h}$ ). Together, these experiments suggest that there may be a synergistic or additive effect on synaptogenesis by inhibiting $\mathrm{HDAC1},-2$, and -3 simultaneously, and that despite its ability to enhance memory when administered to WT mice at the time of training (Malvaez et al, 2013), HDAC3 inhibition alone is insufficient to drive synaptogenesis in this reporter assay.

Prior work has shown that TSA stimulates an increase in the number of presynaptic release sites that correlate with an enhancement in synaptic function in developing neuronal cultures (Akhtar et al, 2009). This is most likely related to the increase in mature synapses induced by the compound. Therefore, we next sought to identify an association between increased synapse number in the synaptogenesis assay and the presence of functional synapses (Figure 3a). We first confirmed the prior report that TSA increases mEPSC frequency $\left(t_{(15)}=2.17, P<0.05\right)$, but not amplitude $(P>0.05)$, relative to DMSO (Figure 3b; Akhtar et al, 2009). Importantly, we observed nearly identical results with RGFP963 treatment (frequency: $t_{(31)}=3.06, P<0.005$, amplitude: $P>0.05$; Figure $3 c$ ), suggesting that the increased SYNYFP+ puncta produced by HDAC1, -2 , and -3 inhibitors (Figure 2) reflects an increase in functional synapses. Also consistent with the results of the synaptogenesis assay, RGFP966 had no effect on the maturation of synaptic function $\left(t_{(17)}=0.71, P>0.05\right.$; Figure $\left.3 \mathrm{~d}\right)$. These data suggest that selective inhibition of HDAC3 is a weak modulator of synaptic function compared with more broad-spectrum class I inhibitors that hit multiple isoforms.

Repeated, daily injections of SAHA, an inhibitor of class I HDACs $(-1,-2,-3$, and -8$)$ and HDAC6, increase the number of dendritic spines in hippocampal Area CA1 in adult mice (Guan et al, 2009). The reported increase in dendritic spine number was accompanied by similar changes in SYN expression, indicating that HDACi-induced spinogenesis enhances network connectivity by stimulating the de novo formation of new synapses in the mature brain. To determine whether HDAC inhibition limited to HDAC1, -2 , and -3 , or HDAC3 alone, was also capable of inducing spinogenesis in vivo, we treated adult Thy1-GFP(m) mice with daily IP injections of either RGFP963 or RGFP966 for 12 days (Figure $4 \mathrm{a}$ ), both of which are brain penetrant (Bowers et al, 2015; Malvaez et al, 2013). The Thy1-GFP(m) line of mice is an excellent tool for determining effects of compounds on spine properties due to the sparse, but intense, expression of eGFP in subsets of pyramidal neurons that results in a Golgi-like quality of neuronal labeling (Feng et al, 2000). RGFP963 increased the density of dendritic spines in CA1 compared with mice treated with vehicle 
a

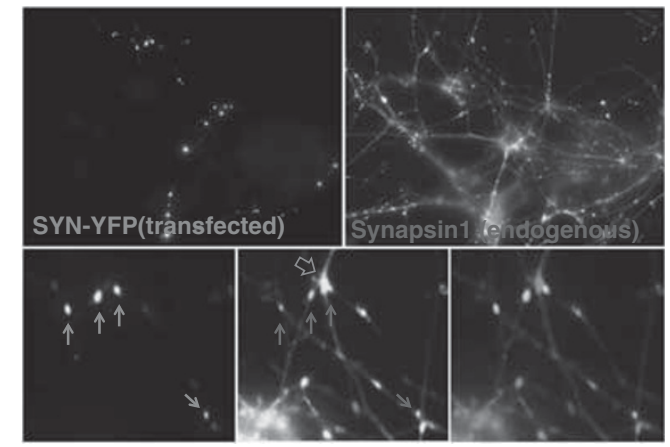

b

Live-neuron synaptogenesis assay

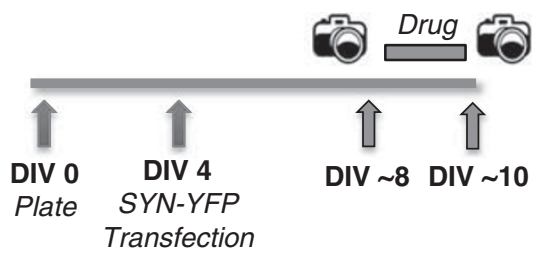

C

DMSO
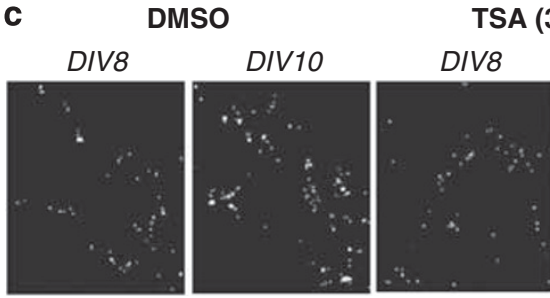

TSA (300 nM)

d

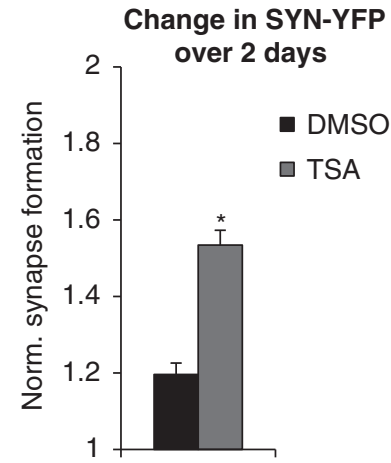

e 963 Dose Response

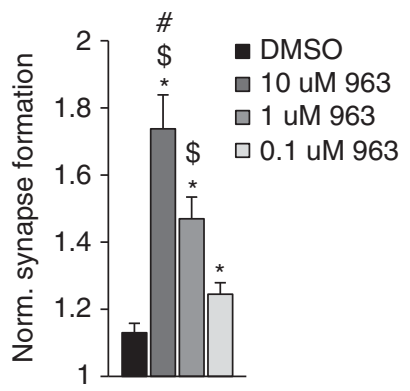

f 966 Dose Response

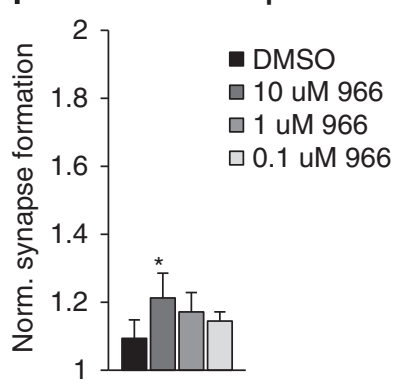

g 233 Dose Response

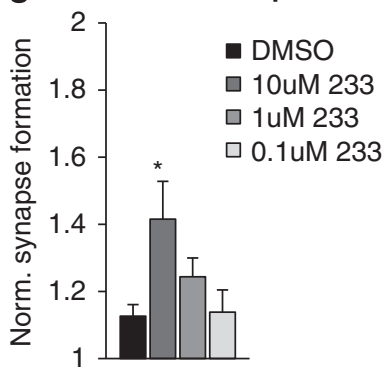

h $10 \mu \mathrm{M}$ HDACi's $=\mathrm{DMSO}$

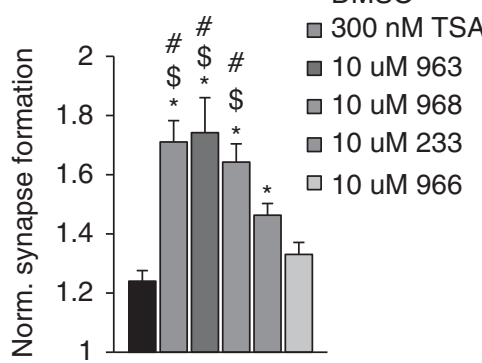

Figure 2 The inhibition of distinct class I histone deacetylases (HDACs) has differential effects on synaptogenesis. (a) Representative images of synaptophysin (SYN)-YFP-transfected neurons. Arrows point to SYN-YFP (green) and synapsin I (red) puncta, respectively. (b) Schematic time line of experiment. Camera symbol indicates when the neurons were imaged. (c) Representative images of SYN-YFP-transfected cortical neurons treated with DMSO or trichostatin A (TSA; $N=6$ per group). (d) Normalized increase of synapse formation from DIV8 to DIVI 0 with TSA or DMSO treatment. Student's $t$-test, $* P<4 \mathrm{E}-5$. (e) Normalized dose response of cortical neurons treated with RGFP963 or DMSO ( $N=6$ per group). One-way ANOVA, * $P<0.05$ relative to DMSO, ${ }^{\$} P<0.05$ relative to $0.1 \mu$ M RGFP963, ${ }^{\#} P<0.05$ relative to I $\mu$ M RGFP963. (f) Normalized dose response to treatment with RGFP966 or DMSO ( $N=6$ per group). One-way ANOVA, *P<0.05. (g) Normalized dose response to treatment with RGFP233 or DMSO ( $N=6$ per group). One-way ANOVA, $* P<0.05$. (h) Relative increase of synapse formation after treatment at maximum efficacy $(T S A=300 \mathrm{nM}$, RGFP compounds $=10 \mu \mathrm{M}$. One-way ANOVA, $* P<0.05$ relative to DMSO, ${ }^{\#} P<0.05$ relative to RGFP233, and ${ }^{\$} P<0.05$ relative to RGFP966. A Tukey HSD post hoc test was applied where appropriate. Error bars reflect SEM. A full colour version of this figure is available at the Neuropsychopharmacology journal online.

$\left(t_{(8)}=-2.87, P<0.05\right)$ (Figure $\left.4 \mathrm{~b}\right)$. Importantly, these results are consistent with prior reports demonstrating an increase in de novo synaptogenesis after treatment with non-specific HDACi's. In contrast, RGFP966 had no effect on spine density when compared with controls ( $P>0.05$; Figure $4 \mathrm{c})$. The lack of an effect with RGFP966 cannot be attributed to poor brain penetrance, as the dose $(30 \mathrm{mg} / \mathrm{kg})$ was three times higher than a dose $(10 \mathrm{mg} / \mathrm{kg})$ that was reported to 
a

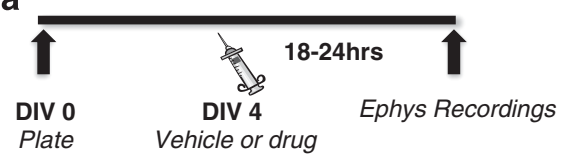

b

DMSO
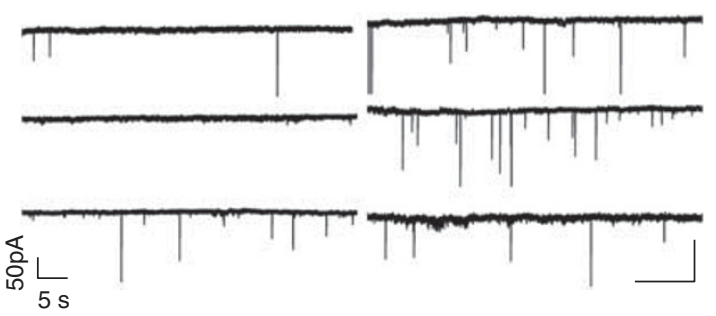

C

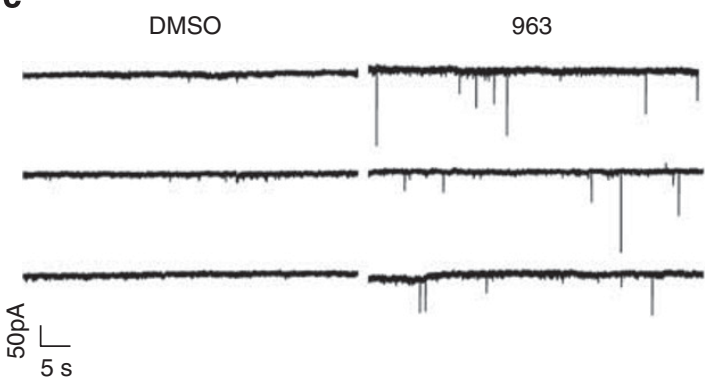

d

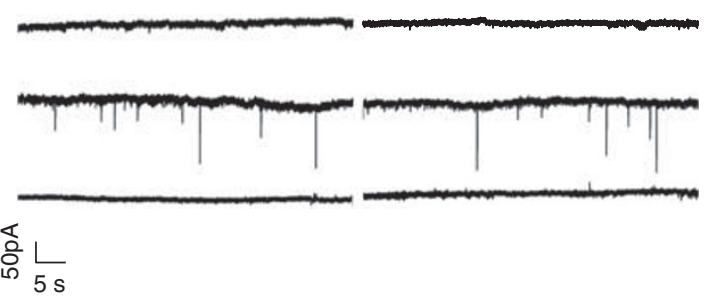

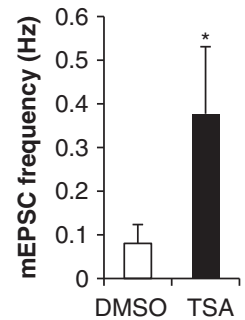
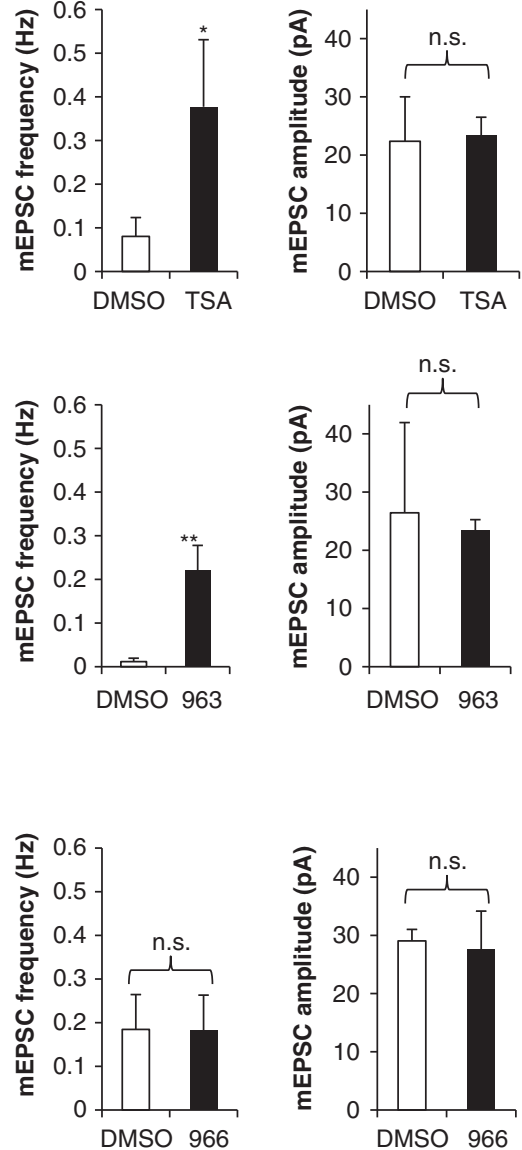

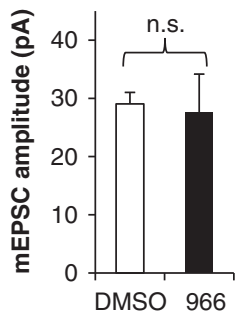

Figure 3 The inhibition of distinct class I histone deacetylases (HDACs) has differential effects on spontaneous excitatory neurotransmission. (a) Schematic time line of experiment. (b) Representative recordings of miniature excitatory events and mean miniature excitatory postsynaptic current (mEPSC) frequency and amplitude in DMSO- $(N=10)$ and TSA $(N=7)$-treated neurons. Student's $t$-test, $* P<0.05$. (c) Representative recordings of miniature excitatory events and mean mEPSC frequency and amplitude in DMSO- $(N=14)$ and RGFP963 $(N=19)$-treated neurons. Student's $t$-test, ${ }^{* * *} P<0.005$. (d) Representative recordings of miniature excitatory events and mean mEPSC frequency and amplitude in DMSO- $(N=10)$ and RGFP966 $(N=9)$-treated neurons. Error bars reflect SEM.

have excellent brain penetrance, reaching maximum concentration within $30 \mathrm{~min}$ of systemic administration. Further, both 10 and $30 \mathrm{mg} / \mathrm{kg}$ doses have been shown to enhance object memory when delivered acutely to adult mice of a similar genetic background (Malvaez et al, 2013).

Considering the relatively distinct selectivity profiles of these compounds, combined with the known impact of HDACi on transcriptionally permissive histone acetylation, we tested the idea that compounds with different class I HDAC selectivity would activate distinct transcriptional patterns in the adult hippocampus. Mice received IP injections of RGFP963, RGFP966, or vehicle at the same doses used for in vivo spine analysis for three consecutive days, and then the dorsal hippocampus was isolated for whole-genome RNA sequencing. The 3-day time point was selected in order to identify transcriptional changes likely to be contributing to development of the new spine formation observed when tissue was collected after 12 days of treatment in the prior experiment. Although both compounds target HDAC3, a relatively small overlapping group of differentially expressed genes was observed ( 20\%; Figure 4d), indicating that combinatorial inhibition of HDAC isoforms may target unique gene groups. We further probed this notion by performing Ingenuity pathway analysis (IPA). Surprisingly, RGFP963 regulated fewer genes than the more selective inhibitor, RGFP966 (Figure 4d). Despite activating fewer genes, IPA identified a greater number of canonical pathways as significantly regulated by RGFP963 than RGFP966. These pathways include 11 associated with synaptogenesis and synaptic function, such as ErbB Signaling, GNRH Signaling, and Synaptic Long Term Potentiation (Figure 4e and Supplementary Table S2A). IPA identified just one 
a

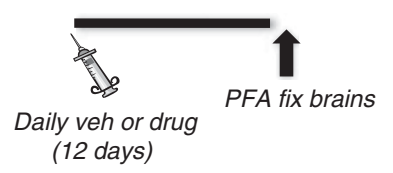

b
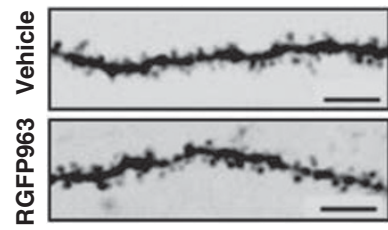

Spine density

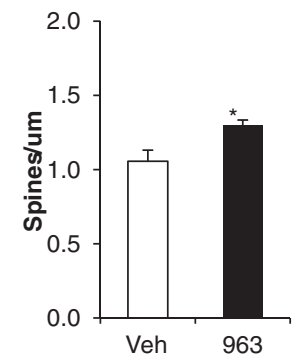

C
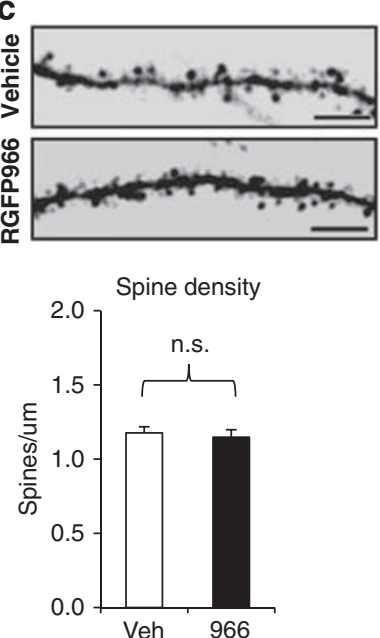

d

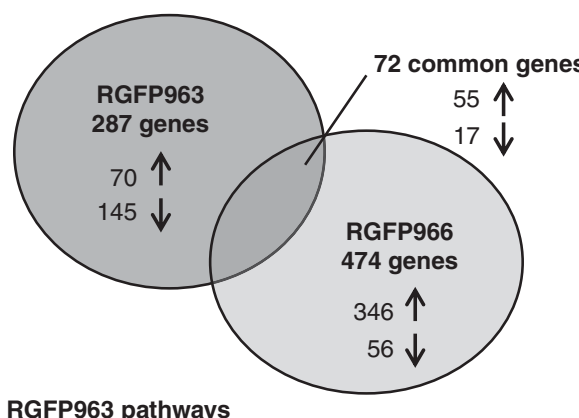

e

RGFP963 pathways

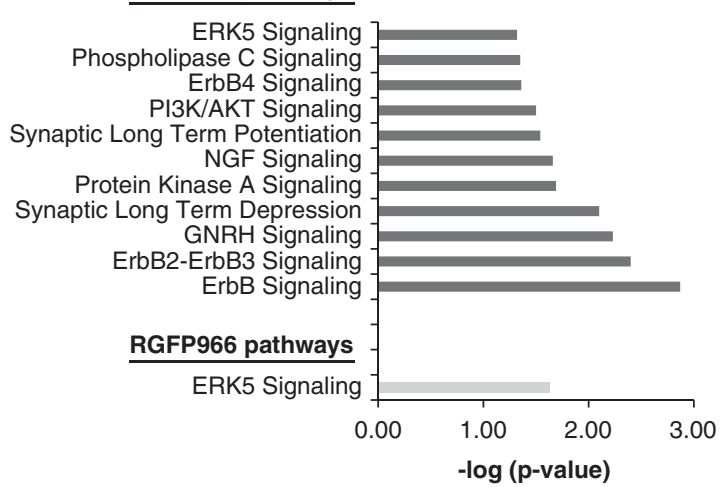

f
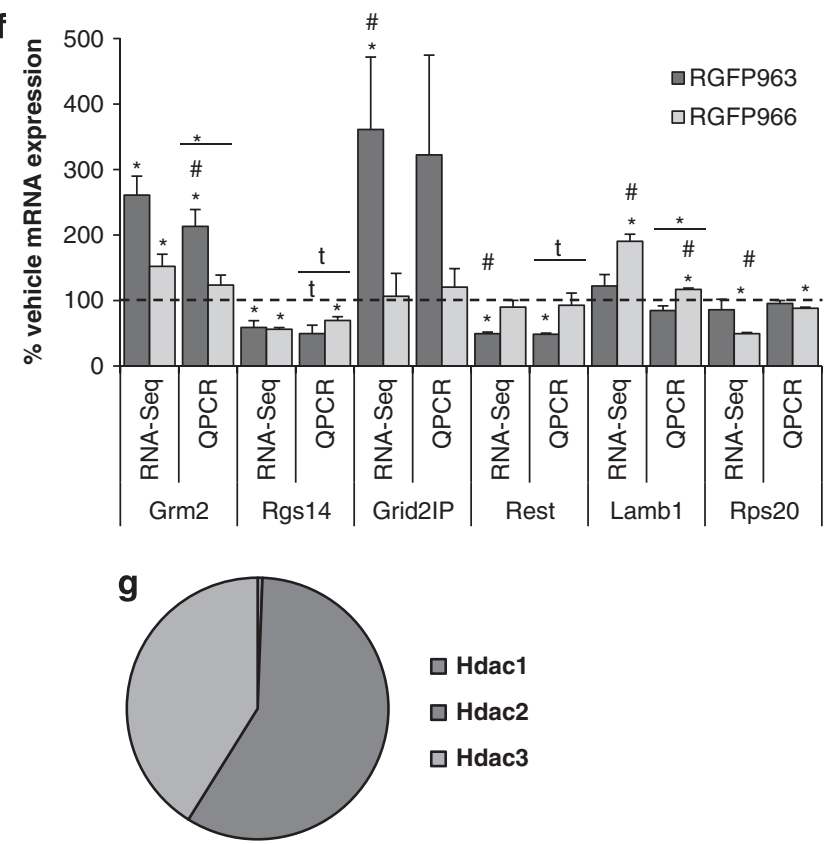

$\square$ Hdac1

$\square$ Hdac2

$\square$ Hdac3

Figure 4 Simultaneous inhibition of histone deacetylase (HDAC)-I, -2, and -3 increased spine density in CAI pyramidal neurons and activated a transcriptional program supportive of synaptogenesis. (a) Schematic time line of experiment. (b) Representative images of dendritic spines from Thy I-GFP( $m$ ) neurons from CAI region of hippocampus and mean spine density following RGFP963 treatment ( $N=5$ per group). (c) Representative images of dendritic spines and mean spine density following RGFP966 treatment. Student's t-test, $* P<0.05$. (d) Systemic injection of RGFP963 and RGFP966 induces both overlapping and unique expression patterns in the dorsal hippocampus. Roughly $20 \%$ of regulated genes were shared in both treatment groups. $N=3$ per group. (e) Predicted pathways with synapse-related functions from the RGFP963- and RGFP966-regulated transcriptomes. ( $f$ ) A subset of genes were selected for GPCR validation and their expression patterns closely mirrored RNA-seq findings. $* P<0.05$, one-way ANOVA comparing vehicle, RGFP963, and RGFP966 groups. *P<0.05, one-tailed $t$-test between vehicle group and RGFP963 or RGFP966 group. ${ }^{*}<<0.05$, two-tailed $t$-test between RGFP963 and RGFP966 groups. $t$ indicates trend $(0.05<P<0.10)$. $N=3$ per group, \pm SEM. (g) Relative expression of the class I HDAC isoforms in dorsal hippocampus tissue. Error bars reflect SEM.

synapse-associated pathway with genes upregulated by RGFP966, ERK5 signaling, which was also identified for RGFP963. The complete list of differentially regulated transcripts and significantly enriched pathways from both treat- ment groups can be found in the Supplementary Material. Validation of the sequencing data was performed for six genes using $\mathrm{qPCR}$ and the results closely matched the RNA-seq patterns (Figure $4 \mathrm{f}$ ). The sequencing data also 
indicated that basal levels of HDAC1 are far lower than HDAC2 and -3 (Figure 4g), suggesting that the majority of RGFP963's effects may be mediated by the latter two isoforms. Consistent with the previous experiment results, these data suggest that RGFP963 may support a more focused transcriptional program supportive of synaptic function.

Enhanced synaptogenesis, leading to improved network power, has been proposed as one of the possible neurobiological substrates contributing to the pro-cognitive effects of HDACi's in models of memory disorders (Fischer et al, 2007). Considering our findings that RGFP963 and RGFP966 have distinct effects on synaptic efficacy and spinogenesis, we next assessed the impact of each compound on cognitive ability in a mouse model of AD. Importantly, the performance of selective HDAC3 inhibitors in models of reduced cognition remains untested. Using the same injection protocol that increased hippocampal spine density with RGFP963 (Figure 4b) and improved memory in several models of memory disruptions with less specific HDACi's (Fischer et al, 2007; Kilgore et al, 2010), we treated 6-monthold APP/PS1 mice and then trained them for contextual fear memory (Figure 5a). Unlike past experiments that have been performed in WT mice in which HDACs are acutely inhibited with a single injection at the time of training, the APP/PS1 mice were drug-free for a minimum of $24 \mathrm{~h}$ before training. This was done in order to focus on the long-term effects of HDAC inhibition, rather than the acute transcriptional effects. One day later, mice were reexposed to the training context to assess long-term contextual memory. Consistent with our previously reported findings (Kilgore et al, 2010), APP/PS1 double-transgenic mice demonstrated a memory deficit relative to WT littermates (Figure 5b and c). We detected a drug-genotype interaction following treatment with RGFP963, indicating that concurrent inhibition of HDAC1, -2, and -3 influenced memory $\left(F_{(1,29)}=9.19, P<0.005\right)$. Post hoc comparisons determined that RGFP963 completely restored memory in the APP/PS1 mice (Figure $5 \mathrm{~b}$ ), a finding consistent with our prior studies in the same mouse line treated with more broad-spectrum HDACi's (Kilgore et al, 2010). The lack of an effect in WT controls is likely due to the chronic treatment regimen used. In separate cohorts of mice, we repeated this experimental design with a dose of RGFP966 that promotes object memory in WT mice (Malvaez et al, 2013). Again, a genotype effect was found $\left(\mathrm{F}_{(1,56)}=37.1, P<1 \mathrm{E}-7 ; \mathrm{N}=15\right.$ per group) indicating that there was a memory disruption in the AD model mice compared with WTs. Interestingly, we did not observe a drug-genotype interaction in this fear conditioning test, which indicates that RGFP966 did not improve memory in this $\mathrm{AD}$ model $(P>0.05)$ (Figure $5 \mathrm{c}$ ).

\section{DISCUSSION}

Using several novel pimelic diphenylamides with differential selectivity for class I HDACs and immediate, long-lasting effects on histone acetylation (Malvaez et al, 2013; Wells et al, 2013; Bowers et al, 2015), we have demonstrated that simultaneous inhibition of HDAC1, -2 , and -3 with RGFP963 drove enhanced synapse function in cultured neurons on par with the pan-HDAC inhibitor, TSA. Interestingly, inhibition a
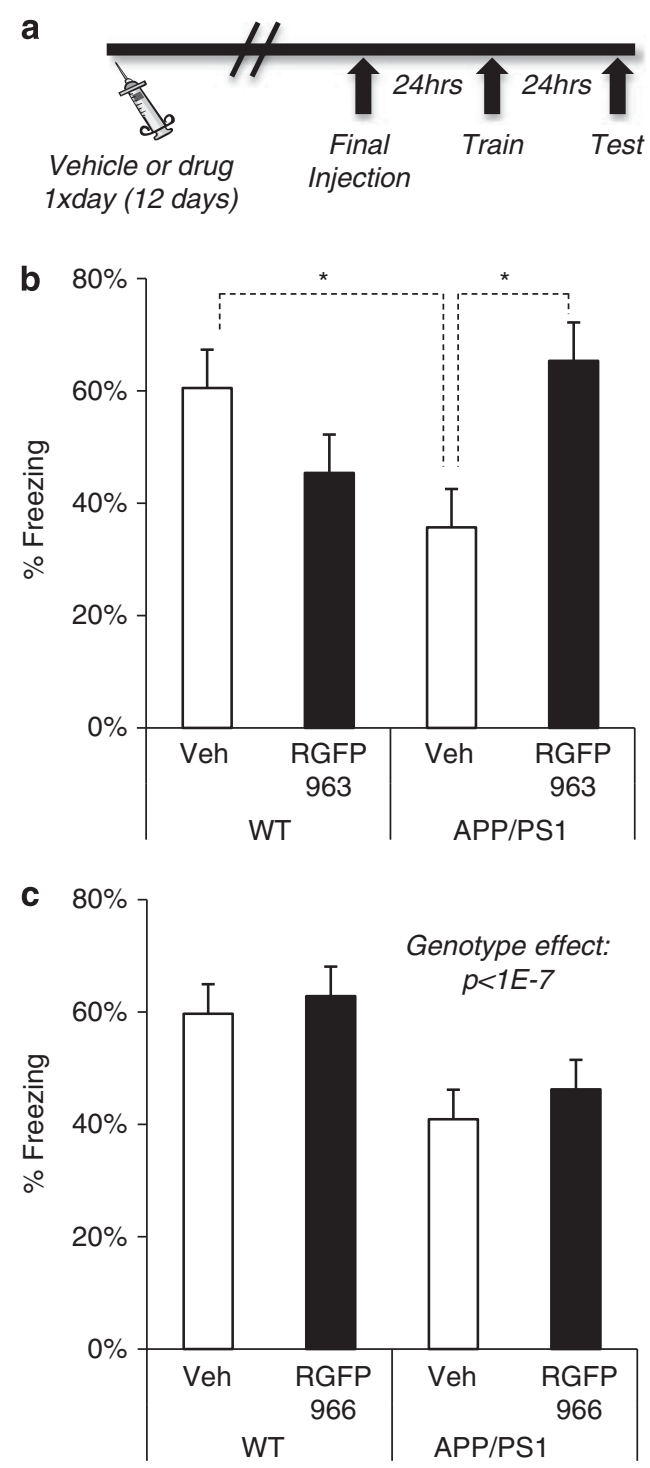

Figure 5 Simultaneous inhibition of histone deacetylase (HDAC)- I, -2, and -3 , but not HDAC3 alone, rescued memory deficits in APPswe/PS I dE9 mice. (a) Schematic time line of experiment. (b) Freezing behavior of mice treated with vehicle (WT: $N=10$, APP/PSI: $N=6$ ) or RGFP963 (WT: $N=8$, APP/PSI: $N=7$ ). (c) Freezing behavior of mice treated with vehicle (WT: $N=15$, APP/PSI: $N=15$ ) or RGFP966 (WT: $N=15$, APP/PSI: $N=15)$. Two-way ANOVA analysis with Fischer LSD post hoc, $* P<0.01$. Error bars reflect SEM.

restricted to HDAC1 and -2 produced less synaptogenesis, with little or no formation of new synapses following treatment with the HDAC3 selective inhibitor, RGFP966. In accordance with the in vitro findings, in vivo inhibition of HDAC1, -2, and -3, but not HDAC3 alone, through systemic delivery of the RGFP compounds increased hippocampal spine density. Unexpectedly, RGFP963 had a more limited impact on transcription than RGFP966. However, bioinformatics analyses indicated that the genes regulated by RGFP963 are predicted to be supportive of enhanced synaptic function. Future studies will be required to provide more firm mechanistic insight into the contribution of these transcriptional changes to the structural plasticity and memory rescue reported here. For example, assessing 
the spinogenic effects of RGFP963 will determine whether this HDAC inhibitor, similar to the less-selective $\mathrm{NaB}$ and SAHA compounds, is able to drive the formation of new spines in the degenerating hippocampus. Finally, RGFP963, but not RGFP966, restored memory in a mouse model of AD.

Given that pharmacological and genetic inhibition of HDAC3 has been shown to enhance object memory in the adult, WT brain (Malvaez et al, 2013; McQuown et al, 2011; Rogge et al, 2013), the lack of an effect of the same dose of RGFP966 on memory in the APP/PS1 model was somewhat surprising. One explanation may lie in the specific behavioral task used or in the timing of treatment. HDAC3 has been shown to enhance hippocampal-based object recognition and location memory tasks, as well as drug-associated memories in healthy animals (Malvaez et al, 2013; Malvaez et al, 2010; Rogge et al, 2013), whereas the current study used contextual fear conditioning in a model of reduced cognitive ability (APP/PS1 mice). It is possible that contextual fear is less dependent on HDAC3, or that HDAC3 inhibition in other brain areas is mediating the cognitive enhancement in object memory tasks. In terms of timing, APP/PS1 mice were given $>24 \mathrm{~h}$ for the RGFP compounds to clear before the start of behavioral training. This design was used in order to focus on longer-term effects of chronic HDACi treatment, rather than the acute transcriptional impact. Further, chronic exposure to HDACi's is more clinically relevant to a therapeutic model of a memory disorder. In other pharmacological tests of HDAC3's contribution to memory, the enzyme was inhibited only once, at the time of training. Therefore, RGFP966 might be capable of promoting memory in APP/PS1 mice if training were performed at the peak of HDAC3 inhibition. A related possibility is that RGFP963's successful memory restoration in APP/PS1 mice may be attributed to a transcriptional priming effect on the promoters of learning-related genes that does not occur with HDAC3 inhibition alone.

In addition to the possibilities just raised, the results of this study may point to something more fundamental. They may indicate that different neurobiological requirements exist for cognitive enhancement in a healthy brain $v s$ memory rescue in a brain with altered network function, similar to that observed in AD models (Abel and Zukin, 2008). In the face of pervasive disruptions to synapse efficacy it is possible that network function must be amplified in order for the brain to support memory storage. Indeed, AD model mice have fewer spines, reduced network activity, and altered memory capacity (Spires-Jones and Knafo, 2012). Disruptions to network capability could, in theory, be restored through enhanced synaptogenesis (Fischer et al, 2007). This is consistent with synapse loss being one of the first structural indicators of AD (Terry et al, 1991). Thus, inhibition of HDAC1, -2 , and -3 may rescue memory in the face of $\mathrm{A} \beta$ induced network disruptions because of an ability to enhance network power through improved synapse efficacy. Interestingly, memory was not enhanced in WT animals by chronic pretreatment with HDAC inhibitors, suggesting that increasing synapse efficacy above baseline levels in a healthy brain does not necessarily lead to memory enhancement.

The current findings highlight simultaneous inhibition of HDAC1, -2 , and -3 as a promising HDAC-based therapy for memory rescue in $\mathrm{AD}$. Although $\mathrm{HDAC1}$ is predominantly expressed in glia and neuronal progenitor cells, HDAC2 is expressed in mature neurons, as is HDAC3 at particularly high levels (Broide et al, 2007; Guan et al, 2009; MacDonald and Roskams, 2008). Although HDAC1 mRNA levels were detected at very low levels compared with HDAC2 and -3, one cannot rule out the possibility that HDAC1 enzymatic activity still contributes to the synaptogenesis observed with the HDAC1 and -2 inhibitor. Although the increased efficacy of the HDAC1, -2 , and -3 inhibitor suggests that simultaneous inhibition of these class I HDAC isoforms might produce a transcriptional synergy that efficiently creates new synapses to rescue memory, it will be important for future studies to compare, side by side, the synaptogenic and cognitive-enhancing properties of an HDAC1, -2, and -3 inhibitor to that of HDAC2, -3 and selective HDAC2 inhibitors (Wagner et al, 2015) to determine the optimal balance between efficacy and off-target effects in relevant disease models.

\section{FUNDING AND DISCLOSURE}

GR received financial support for this project from Repligen Corporation. The remaining authors declare no conflict of interest.

\section{ACKNOWLEDGMENTS}

We would like to thank Mohammad Fallahi of the Scripps Florida Bioinformatics Core for his assistance with the IPA analysis. This work was supported by grants to CAM from The National Institute on Drug Abuse (R01DA034116 and R03DA033499); to GR from The National Institute for Neurological Disorders and Stroke (R01NS064079 and R21NS082640), The National Institute for Mental Health (R01MH096847), and Repligen Corporation; and to JDS from the National Institute for Mental Health (R01MH57014).

\section{REFERENCES}

Abel T, Zukin RS (2008). Epigenetic targets of HDAC inhibition in neurodegenerative and psychiatric disorders. Curr Opin Pharmacol 8: 57-64.

Akhtar MW, Raingo J, Nelson ED, Montgomery RL, Olson EN, Kavalali ET et al (2009). Histone deacetylases 1 and 2 form a developmental switch that controls excitatory synapse maturation and function. J Neurosci 29: 8288-8297.

Blank M, Dornelles AS, Werenicz A, Velho LA, Pinto DF, Fedi AC et al (2014). Basolateral amygdala activity is required for enhancement of memory consolidation produced by histone deacetylase inhibition in the hippocampus. Neurobiol Learn Mem 111: 1-8.

Bowers ME, Xia B, Carreiro S, Ressler KJ (2015). The Class I HDAC inhibitor RGFP963 enhances consolidation of cued fear extinction. Learn Mem 22: 225-231.

Broide RS, Redwine JM, Aftahi N, Young W, Bloom FE, Winrow CJ (2007). Distribution of histone deacetylases 1-11 in the rat brain. J Mol Neurosci 31: 47-58.

Day JJ, Sweatt JD (2011). Epigenetic mechanisms in cognition. Neuron 70: 813-829.

Fass DM, Reis SA, Ghosh B, Hennig KM, Joseph NF, Zhao WN, Nieland TJ et al (2013). Crebinostat: a novel cognitive enhancer that inhibits histone deacetylase activity and modulates chromatinmediated neuroplasticity. Neuropharmacology 64: 81-96. 
Feng G, Mellor RH, Bernstein M, Keller-Peck C, Nguyen QT, Wallace $M$ et al (2000). Imaging neuronal subsets in transgenic mice expressing multiple spectral variants of GFP. Neuron 28: 41-51.

Fischer A, Sananbenesi F, Wang X, Dobbin M, Tsai LH (2007). Recovery of learning and memory is associated with chromatin remodelling. Nature 447: 178-182.

Graff J, Rei D, Guan JS, Wang WY, Seo J, Hennig KM et al (2012). An epigenetic blockade of cognitive functions in the neurodegenerating brain. Nature 483: 222-226.

Graff J, Tsai LH (2013). The potential of HDAC inhibitors as cognitive enhancers. Annu Rev Pharmacol Toxicol 53: 311-330.

Guan JS, Haggarty SJ, Giacometti E, Dannenberg JH, Joseph N, Gao J et al (2009). HDAC2 negatively regulates memory formation and synaptic plasticity. Nature 459: 55-60.

Haggarty SJ, Tsai LH (2011). Probing the role of HDACs and mechanisms of chromatin-mediated neuroplasticity. Neurobiol Learn Mem 96: 41-52.

Harms KJ, Tovar KR, Craig AM (2005). Synapse-specific regulation of AMPA receptor subunit composition by activity. J Neurosci 25: 6379-6388.

Hawk JD, Florian C, Abel T (2011). Post-training intrahippocampal inhibition of class I histone deacetylases enhances long-term object-location memory. Learn Mem 18: 367-370.

Herman D, Jenssen K, Burnett R, Soragni E, Perlman SL, Gottesfeld JM (2006). Histone deacetylase inhibitors reverse gene silencing in Friedreich's ataxia. Nat Chem Biol 2: 551-558.

Intlekofer KA, Berchtold NC, Malvaez M, Carlos AJ, McQuown SC, Cunningham MJ et al (2013). Exercise and sodium butyrate transform a subthreshold learning event into long-term memory via a brain-derived neurotrophic factor-dependent mechanism. Neuropsychopharmacology 38: 2027-2034.

Jankowsky JL, Xu G, Fromholt D, Gonzales V, Borchelt DR (2003). Environmental enrichment exacerbates amyloid plaque formation in a transgenic mouse model of Alzheimer disease. J Neuropathol Exp Neurol 62: 1220-1227.

Jia H, Kast RJ, Steffan JS, Thomas EA (2012). Selective histone deacetylase (HDAC) inhibition imparts beneficial effects in Huntington's disease mice: implications for the ubiquitin-proteasomal and autophagy systems. Hum Mol Genet 21: 5280-5293.

Kilgore M, Miller CA, Fass DM, Hennig KM, Haggarty SJ, Sweatt JD et al (2010). Inhibitors of class 1 histone deacetylases reverse contextual memory deficits in a mouse model of Alzheimer's disease. Neuropsychopharmacology 35: 870-880.

Knobloch M, Mansuy IM (2008). Dendritic spine loss and synaptic alterations in Alzheimer's disease. Mol Neurobiol 37: 73-82.

Levenson JM, O'Riordan KJ, Brown KD, Trinh MA, Molfese DL, Sweatt JD (2004). Regulation of histone acetylation during memory formation in the hippocampus. J Biol Chem 279: 40545-40559.

MacDonald JL, Roskams AJ (2008). Histone deacetylases 1 and 2 are expressed at distinct stages of neuro-glial development. Dev Dyn 237: 2256-2267.

Mahan AL, Mou L, Shah N, Hu JH, Worley PF, Ressler KJ (2012). Epigenetic modulation of Homerla transcription regulation in amygdala and hippocampus with pavlovian fear conditioning. J Neurosci 32: 4651-4659.
Malvaez M, McQuown SC, Rogge GA, Astarabadi M, Jacques V, Carreiro S et al (2013). HDAC3-selective inhibitor enhances extinction of cocaine-seeking behavior in a persistent manner. Proc Natl Acad Sci USA 110: 2647-2652.

Malvaez M, Sanchis-Segura C, Vo D, Lattal KM, Wood MA (2010). Modulation of chromatin modification facilitates extinction of cocaine-induced conditioned place preference. Biol Psychiatry 67: 36-43.

McQuown SC, Barrett RM, Matheos DP, Post RJ, Rogge GA, Alenghat $\mathrm{T}$ et al (2011). HDAC3 is a critical negative regulator of long-term memory formation. J Neurosci 31: 764-774.

Mikaelsson MA, Miller CA (2011). The path to epigenetic treatment of memory disorders. Neurobiol Learn Mem 96: 13-18.

Monsey MS, Ota KT, Akingbade IF, Hong ES, Schafe GE (2011). Epigenetic alterations are critical for fear memory consolidation and synaptic plasticity in the lateral amygdala. PloS One 6: e19958.

Morris MJ, Mahgoub M, Na ES, Pranav H, Monteggia LM (2013). Loss of histone deacetylase 2 improves working memory and accelerates extinction learning. J Neurosci 33: 6401-6411.

Nistico R, Pignatelli M, Piccinin S, Mercuri NB, Collingridge G (2012). Targeting synaptic dysfunction in Alzheimer's disease therapy. Mol Neurobiol 46: 572-587.

Pozueta J, Lefort R, Shelanski ML (2013). Synaptic changes in Alzheimer's disease and its models. Neuroscience 251: 51-65.

Rai M, Soragni E, Jenssen K, Burnett R, Herman D, Coppola G et al (2008). HDAC inhibitors correct frataxin deficiency in a Friedreich ataxia mouse model. PloS One 3: e1958.

Ricobaraza A, Cuadrado-Tejedor M, Perez-Mediavilla A, Frechilla D, Del Rio J, Garcia-Osta A (2009). Phenylbutyrate ameliorates cognitive deficit and reduces tau pathology in an Alzheimer's disease mouse model. Neuropsychopharmacology 34: 1721-1732.

Rogge GA, Singh H, Dang R, Wood MA (2013). HDAC3 is a negative regulator of cocaine-context-associated memory formation. J Neurosci 33: 6623-6632.

Rudenko A, Tsai LH (2014). Epigenetic modifications in the nervous system and their impact upon cognitive impairments. Neuropharmacology 80C: 70-82.

Shi P, Scott MA, Ghosh B, Wan D, Wissner-Gross Z, Mazitschek R et al (2011). Synapse microarray identification of small molecules that enhance synaptogenesis. Nat Commun 2: 510.

Spires-Jones T, Knafo S (2012). Spines, plasticity, and cognition in Alzheimer's model mice. Neural Plast 2012: 319836.

Terry RD, Masliah E, Salmon DP, Butters N, DeTeresa R, Hill R et al (1991). Physical basis of cognitive alterations in Alzheimer's disease: synapse loss is the major correlate of cognitive impairment. Ann Neurol 30: 572-580.

Thomas EA, Coppola G, Desplats PA, Tang B, Soragni E, Burnett R et al (2008). The HDAC inhibitor $4 \mathrm{~b}$ ameliorates the disease phenotype and transcriptional abnormalities in Huntington's disease transgenic mice. Proc Natl Acad Sci USA 105: 15564-15569.

Wagner FF, Zhang YL, Fass DM, Joseph N, Gale JP, Weiwer M et al (2015). Kinetically selective inhibitors of histone deacetylase 2 (HDAC2) as cognition enhancers. Chem Sci 6: 804-815.

Wells CE, Bhaskara S, Stengel KR, Zhao Y, Sirbu B, Chagot B et al (2013). Inhibition of histone deacetylase 3 causes replication stress in cutaneous T cell lymphoma. PLoS One 8: e68915.

Supplementary Information accompanies the paper on the Neuropsychopharmacology website (http://www.nature.com/npp) 